\title{
BMJ Open Protocol for developing a mental imagery intervention: a randomised controlled trial testing a novel implementation imagery e-health intervention to change driver behaviour during floods
}

Kyra Hamilton, ${ }^{1,2}$ Jacob J Keech, ${ }^{1}$ Amy E Peden, ${ }^{3,4}$ Martin S Hagger ${ }^{2,5}$

To cite: Hamilton K, Keech JJ, Peden AE, et al. Protocol for developing a mental imagery intervention: a randomised controlled trial testing a novel implementation imagery e-health intervention to change driver behaviour during floods. BMJ Open 2019;9:e025565. doi:10.1136/ bmjopen-2018-025565

- Prepublication history and additional material for this paper are available online. To view these files, please visit the journal online (http://dx.doi org/10.1136/bmjopen-2018025565).

Received 23 July 2018 Revised 16 November 2018 Accepted 18 December 2018
D) Check for updates

(C) Author(s) (or their employer(s)) 2019. Re-use permitted under CC BY-NC. No commercial re-use. See rights and permissions. Published by BMJ.

For numbered affiliations see end of article.

Correspondence to

Dr Kyra Hamilton;

kyra.hamilton@griffith.edu.au

\section{ABSTRACT}

Introduction Drowning due to driving into floodwater accounts for a significant proportion of all deaths by drowning. Despite awareness campaigns such as 'If it's flooded, forget it', people continue to drive into floodwater. This causes loss of life, risk to rescuers and damage to vehicles. The aim of this study was to develop and evaluate an online e-health intervention to promote safe driving behaviour during flood events.

Methods and analysis The study will use a $2 \times 3$ randomised controlled trial in which participants are randomised into one of two conditions: (1) education about the risks of driving into floodwater or (2) education about the risks of driving into floodwater plus a theory-based behaviour change intervention using planning and imagery exercises. The effect of the intervention on the primary outcome, intention to drive through floodwater and the secondary outcomes will be assessed using a series of mixed-model analysis of covariances.

Ethics and dissemination The study has been approved by the Griffith University Human Research Ethics Committee. Participants will review a study information sheet and provide informed consent prior to commencing participation. Results will be disseminated through peerreviewed publications, industry reports, media releases and at academic conferences. Deidentified data will be made publicly available following publication of the results. Trial registration number ACTRN12618001212246.

\section{INTRODUCTION}

Drowning is the third leading cause of injury-related deaths worldwide ${ }^{1}$ and the leading cause of death during times of flood. ${ }^{23}$ Activities such as driving into, walking near or engaging in recreational activities around or in floodwater are commonly reported as preceding drowning. ${ }^{1}$ Reports have shown that, in Australia, around 53\% of flood-related deaths and $55 \%$ of all river flood-related unintentional fatal deaths ${ }^{4}$ were the
Strengths and limitations of this study

- The study intervention is theory-based, consisting of a novel integration of effective behaviour change strategies.

- The study will use a sample with key demographic characteristics proportional to the distribution of flood-related transport deaths in the Australian population, ensuring that the results are generalisable.

- While the outcomes are an extensive range of psychological variables established to predict driving into floodwater, the unpredictable occurrence of flood events means that examining the effect of our intervention on actual behaviour is not feasible within the timeframe of the study.

result of driving into floodwaters. Another recent study surveyed $>600$ Australian river users and found $36 \%$ of participants reported having driven into floodwater, with males $(44 \%)$ significantly more likely to have driven into floodwaters than females $(28 \%) .^{5}$ These drowning statistics also likely underestimate the true extent of the driving-related drownings due to limitations around the use of International Classification of Diseases flood-related drowning codes. ${ }^{67}$

Due to the magnitude of this issue, intentionally driving into floodwater has been the subject of mass-media drowning prevention campaigns such as 'Turn Around Don't Drown' ${ }^{8}$ in the USA and 'If it's flooded, forget it ${ }^{9}$ in Australia. However, very little research to date has evaluated the effect of these campaigns on attitudes, motivation and actual drowning rates. In fact, fatal and non-fatal drownings resulting from intentionally driving into floodwater continue to occur regularly. ${ }^{10-12}$ For example, during a 
severe weather event in a 24-hour period, in March 2017, 108 floodwater rescues were conducted in Queensland, Australia by the State Emergency Service. ${ }^{13}$ This has resulted in a national call for research into behaviours around floodwater. ${ }^{12}$

In response to a paucity of empirical research, we conducted a series of studies to understand the psychological processes underpinning decisions around driving into floodwater, ${ }^{14-16}$ avoiding driving into floodwater ${ }^{17}$ and the experiences of those who rescue drivers who have driven into floodwater. ${ }^{18}$ While it is commonly assumed that people choose to drive into floodwater due to a lack of awareness of the risks, our research identified that many of the people who drive into floodwater are aware of the risks and have been exposed to relevant massmedia campaigns. ${ }^{14}$

Building on this research, we developed and evaluated a mass-media style infographic video that included theory-based behaviour change methods to bridge the gap between awareness and intentions to drive into floodwater. ${ }^{19}$ While the video had an effect on attitudes, subjective norm, perceived susceptibility and perceived severity immediately postintervention, these effects were only maintained at the 4-week follow-up for women and not men. Furthermore, there was no effect of the video on intentions. ${ }^{19}$ Therefore, continued development and evaluation of interventions aimed at promoting safe driving behaviour during floods is a priority. Some potential explanations for the null effect of the infographic on intentions are that the intervention did not directly target intentions and/or did not aid in the development of a plan that can be implemented if this particular situation arises for the person during a flood event (ie, faced with a flooded road while in their vehicle). We also suspect that gender differences in the effects at follow-up may have been due to females self-reinforcing the messages contained within the intervention due to a greater tendency to use ruminative thinking styles. ${ }^{20}$ This suggests that an effective intervention aimed at promoting safe driving behaviour during floods should use behaviour change methods that aid in the development of a plan and when to implement it, such as implementation intentions ${ }^{21}$; and allow sufficient internalisation of the intervention content through strategies such as mental imagery. ${ }^{22}$

Implementation intentions are concrete plans about when, where and how to enact a behaviour to achieve a specific goal which contrasts from simply intending to achieve a goal-otherwise known as goal intentions. ${ }^{23}$ Implementation intentions have been found to be superior to goal intentions in achieving goals, with a meta-analysis finding medium to large effects of implementation intentions on goal achievement. ${ }^{24}$ Conroy and Hagger ${ }^{22}$ describe mental imagery interventions as involving 'self-directed imagining or visualising specific events, actions or outcomes, including concomitant feelings and responses, with the express purpose of increasing motivation toward a target action or task' (p. 669). The meta-analysis of imagery interventions by Conroy and
Hagger in health behaviour found non-trivial small averaged corrected effects of these interventions on postintervention behaviours, intentions, perceived control and attitudes; and identified characteristics of interventions that moderate the effect size. A small number of studies have combined implementation intentions and mental imagery to change health behaviours such as fruit and vegetable consumption ${ }^{25}$ and alcohol intake ${ }^{26}$ yielding small to medium effect sizes. In building on our prior work, ${ }^{19}$ we therefore seek to combine two behaviour change strategies-mental imagery and implementation intentions-to develop an implementation imagery intervention to promote less favourable intentions to drive into floodwater. Implementation imagery is an intervention procedure comprising imagery and planning exercises. Participants are prompted to imagine the steps required to engage in a future motivated behaviour and form a concrete plan to implement the steps. We propose a six-part implementation imagery intervention procedure for the proposed intervention aimed at changing driver beliefs and intentions to drive into floodwater. In part 1, participants will be provided with information on the target behaviour. In part 2, participants will receive persuasive communication prompting participants to form a goal to perform the target behaviour in the future. In part 3, participants will be asked to complete a practice imagery exercise. In part 4, respondents will be instructed to imagine the steps required for them to achieve the goal set in part 2 and to develop and specify a plan to follow the steps if they were to encounter floodwater while driving. In part 5, participants will be prompted to imagine the outcomes associated with enacting the plan to highlight the personal relevance of the plan. In part 6 , participants will be provided with a final statement indicating when they should remember their plan.

\section{Objectives}

Drawing on our previous research, ${ }^{14-19}$ we aim to develop a theory-based behavioural intervention to promote less favourable intentions of drivers to drive into floodwaters. The research will be conducted in two phases, a development phase in which the theory-based content of the implementation imagery intervention embedded in a survey will be developed and piloted, and an implementation and evaluation phase in which the effect of the intervention in changing intentions and beliefs with respect to driving into floodwaters, will be tested in a sample of drivers using a randomised controlled design. Drivers recruited to the trial are randomly assigned to intervention or control conditions. Participants assigned to the implementation imagery condition receive a set of education-based messages that focus on changing attitudes and intentions with respect to driving into floodwaters, and the theory-based implementation imagery exercises. The control condition receives the education-based messages alone, which are drawn from information commonly disseminated by public safety organisations. This represents a 'usual care' control condition. Specifically, 
the objectives of the current study are to: (a) test the effectiveness of the intervention administered after baseline measures of study constructs at time 1 (T1) in changing drivers' intentions and beliefs immediately postintervention at time 2 (T2); (b) determine whether the effects of the intervention are maintained 4 weeks later at time 3 (T3); compare the effects of the intervention to effects of a comparison condition comprising publicly available education on driving into floodwater and (d) examine sex differences in intervention effects at T2 and T3.

We hypothesise that drivers assigned to the intervention condition will report significantly lower intentions to drive through floodwater, relative to the control condition immediately postintervention at T2 (primary outcome; hypothesis 1). With regard to the secondary outcomes, we hypothesise that drivers assigned to the intervention condition will report significantly less favourable attitudes and reduced perceived social pressure to drive through floodwater, as well as greater perceptions of behavioural control, risk perception, perceived susceptibility, perceived severity and anticipated regret towards driving through floodwater, relative to the control condition immediately postintervention at T2 (hypothesis 2). We further hypothesise that drivers assigned to the intervention condition will report significantly greater barrier self-efficacy and action planning regarding avoiding driving through floodwater, relative to the control condition immediately postintervention at T2 (hypothesis 3 ). We also expect that the effects of the intervention will be maintained 4 weeks later at T3 (hypothesis 4). Given our previous research has identified sex differences in changing beliefs, ${ }^{19}$ we will also test for sex differences in the effects of the intervention on study outcomes.

\section{METHODS AND ANALYSIS}

The study protocol is reported in accordance with Standard Protocol Items: Recommendations for Interventional Trials (SPIRIT) standard protocol items for clinical trials. $^{27} 28$

\section{Intervention development and optimisation}

The intervention content was developed based on existing safety messages, ${ }^{9}$ our prior research, ${ }^{1417}$ behaviour change methods ${ }^{29}$ and best-practice techniques for implementation intentions and mental imagery. ${ }^{22}$ Prior to recording, the scripts were reviewed by a panel of experts in drowning prevention, behaviour change and media and communication. The scripts were also piloted with two Australian drivers and feedback was invited. Based on initial expert and driver feedback, refinements to wording of the scripts were made. The scripts were then audio-recorded using a voiceover actor and developed into videos. The videos then underwent further expert review and piloting with seven drivers from the target population. The pilot involved the participants completing the T1 survey, the intervention, the T2 survey and then a semi-structured interview where they were asked broadly about their thoughts regarding the exercises. The participants were probed for specific information regarding clarity and timing of the different exercises in the video if it was not already shared. The drivers were purposively recruited to ensure a range on key demographic factors of age, sex and education level. Based on qualitative feedback provided by pilot participants, we made data-driven refinements to the presentation and timing allocated to the exercises.

\section{Patient and public involvement}

Patient and public involvement in the development of the intervention was conducted through our prior research which involved qualitative interviews with members of the target population ${ }^{14}{ }^{17}$-Australian drivers-and through piloting of the intervention materials with Australian drivers. Specifically, drivers' descriptions of the behaviour informed the wording of the items used in the outcome measures. Drivers' descriptions from these studies also informed the content of the intervention. The research questions were developed around evaluating the effect of the intervention on psychological constructs that have been found to predict willingness to drive through floodwater in prior research. ${ }^{15} 16$ Drivers/participants will not be involved in the recruitment or conduct of the study. Participants will be provided with the contact details of the research team on the study information sheet and will be informed that they can contact the research team if they wish to receive the results of the study. The burden of participating will be assessed by the drivers after having read the study information and prior to provision of informed consent.

\section{Study design}

The intervention will be evaluated against the control condition immediately postintervention (T2) and 4 weeks later (T3). The evaluation will adopt a 2 (group/condition: education and implementation intention imagery condition vs education only condition) $\times 3$ (time: T1, T2, T3) parallel group randomised controlled design. Figure 1 illustrates the design of the intervention and participant flow through the study.

\section{Setting and participant recruitment}

The randomised controlled trial will be conducted using an online survey tool (Qualtrics). Participants will be eligible if they hold a valid full driver's licence, are an Australian resident and are the only member of their household participating in the study. Participants will be recruited using a research panel provider and screened before entering the trial. In addition to the inclusion criteria, participants will be screened on the following demographic characteristics and quotas will be imposed to ensure that the sample comprises similar proportions of these characteristics to the distribution of flood-related transport deaths in the Australian general population: age, sex, geographic region (by state and metropolitan vs rural) and household income. The study will adopt a double-blind procedure. Participants will be unaware of 


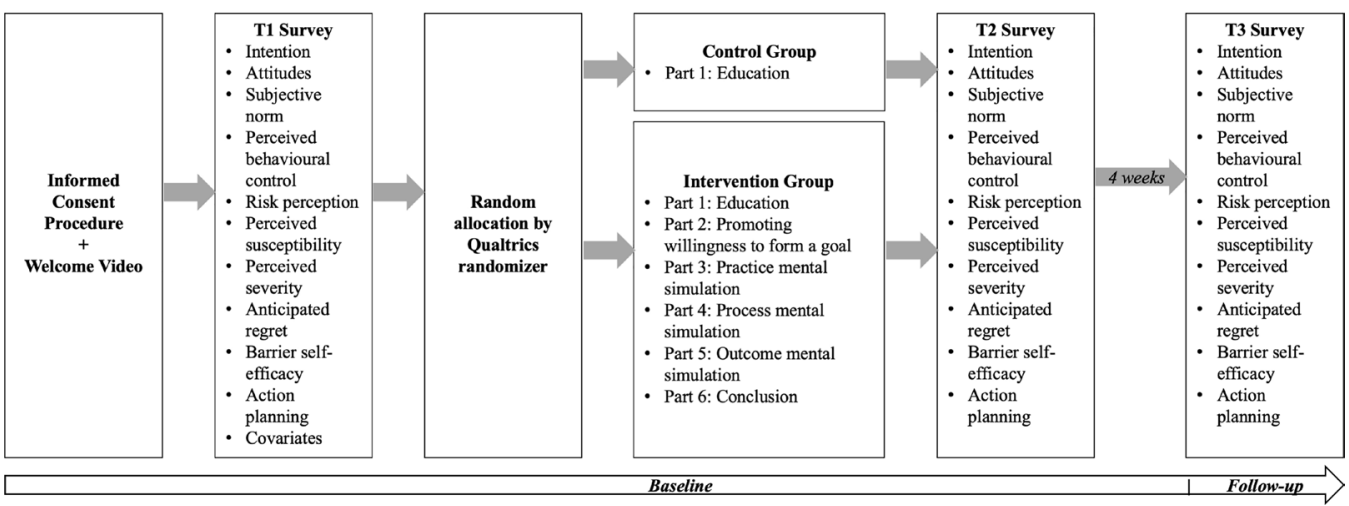

Figure 1 Randomised controlled trial design and participant progression through the study.

the condition to which they have been assigned and study purpose. Staff at the research panel provider company who may have direct contact with the participants will also be unaware of the conditions and purpose of the study.

\section{Data quality}

Two questions will be embedded within the T1 survey to assess attentive responding. ${ }^{3031}$ The questions instruct the choice of a particular answer so that it is not possible to answer the question incorrectly if the item is read carefully (eg, 'please choose option two to ensure you are paying attention'). Participants who do not answer both of the items correctly will be excluded.

\section{Randomisation}

Randomisation into one of the two groups will be conducted by the Qualtrics randomiser feature following the T1 survey. The Qualtrics randomiser operates using a Mersenne Twister pseudorandom number generator. The Mersenne Twister is the default pseudorandom number generator for a range of widely used software packages including Microsoft Excel and SPSS. By nature of this method of random assignment, the sequence cannot be determined until the participant is assigned.

\section{Intervention condition}

Participants will be initially presented with a brief information sheet outlining study expectations and participant rights prior to completing an informed consent form. They will then watch a brief 'welcome' video to familiarise participants with the voice of the narrator, and to give participants the opportunity to enable the audio on their device prior to commencing the intervention. Next, participants will complete the T1 (preintervention) questionnaire, after which they will be randomised into one of two conditions (see figure 1 for trial design). Both groups will watch the welcome video; however, the welcome video for the control group does not make reference to the mental imagery activity. The intervention group will receive the following six-part procedure, while the control group will receive only part 1 . Participants are then directed to the T2 survey (see online supplementary material A for video scripts). The intervention is structured such that participants receive information, then form a goal intention and then go on to make a plan. After imagining their plan and noting it down, participants are instructed to imagine the outcomes associated with enacting their plan to highlight personal relevance. The intervention uses a range of behaviour change methods ${ }^{29}$ which have been mapped on to the theoretical constructs that comprise the study outcomes (table 1).

\section{Part 1: education}

The first video is designed to educate participants on the risks of driving into floodwater. It has been composed based on information that is publicly available and commonly broadcasted in Australia.

\section{Part 2: formation of a goal intention}

The second video is designed to encourage participants to form a goal intention to avoid driving into floodwater if they encounter it on their route and to indicate that by forming a plan of what do in this situation, they can achieve their goal and avoid driving into floodwater. The video contains visuals of rain, floodwater and a floodwater damaged road, and drivers are provided with further information about why people drive into floodwater which is drawn from our prior research. ${ }^{14} 17$ Following part 2, participants will complete a single-item measure of goal intention, indicating their commitment to the goal (see online supplementary material B for question details).

\section{Part 3: practice imagery exercise}

Prior to the process imagery exercise, participants are provided with a practice guided imagery exercise. The purpose of the exercise is to allow participants to familiarise them with the practice of imagery, and to get them to relax and begin visualising vivid images. The practice guided imagery exercise prompts participants to imagine cutting into a lemon with a knife. The exercise uses a familiar situation in order to facilitate imagery.

\section{Part 4: process imagery exercise}

Following the practice exercise, participants are provided with some examples of safe options to take should they encounter floodwater on their route. Participants are then instructed to imagine approaching floodwaters 
Table 1 Behaviour change methods, targeted theoretical constructs and implementation strategies

\begin{tabular}{|c|c|c|c|}
\hline Part & $\begin{array}{l}\text { Behaviour change } \\
\text { method/s }\end{array}$ & Implementation strategy & Target construct \\
\hline $\begin{array}{l}\text { 1: } \\
\text { Education }\end{array}$ & Information provision & $\begin{array}{l}\text { Provide information about the risks of driving } \\
\text { into floodwater }\end{array}$ & $\begin{array}{l}\text { Attitudes, risk perception, perceived } \\
\text { severity, perceived susceptibility }\end{array}$ \\
\hline $\begin{array}{l}\text { 2: } \\
\text { Formation } \\
\text { of a goal } \\
\text { intention }\end{array}$ & $\begin{array}{l}\text { Personalise risk } \\
\text { Scenario-based risk } \\
\text { information } \\
\text { Provide opportunities for } \\
\text { social comparison } \\
\text { Goal setting }\end{array}$ & $\begin{array}{l}\text { Providing information about the personal } \\
\text { risk; providing reasons people commonly } \\
\text { drive into floodwater from prior studies; } \\
\text { providing a strategy for overcoming barriers } \\
\text { to avoiding driving into floodwater }\end{array}$ & $\begin{array}{l}\text { Intention, attitudes, subjective norm, } \\
\text { perceived behavioural control, risk } \\
\text { perception, perceived severity, } \\
\text { perceived susceptibility, anticipated } \\
\text { regret, barrier self-efficacy }\end{array}$ \\
\hline $\begin{array}{l}\text { 3: Practice } \\
\text { imagery } \\
\text { exercise }\end{array}$ & $\begin{array}{l}\text { Guided practice (imagery } \\
\text { skill) }\end{array}$ & Tangy lemon guided imagery task & Not applicable \\
\hline $\begin{array}{l}\text { 4: Process } \\
\text { mental } \\
\text { simulation }\end{array}$ & $\begin{array}{l}\text { Implementation intentions } \\
\text { Goal setting } \\
\text { Planning coping responses } \\
\text { Guided practice } \\
\text { Using imagery }\end{array}$ & $\begin{array}{l}\text { Provide examples of things to do when } \\
\text { floodwater is encountered; imagining the } \\
\text { steps to use when encountering floodwater } \\
\text { while driving; process mental simulation } \\
\text { exercise }\end{array}$ & $\begin{array}{l}\text { Intention, perceived behavioural control, } \\
\text { barrier self-efficacy, action planning }\end{array}$ \\
\hline $\begin{array}{l}\text { 5: } \\
\text { Outcome } \\
\text { mental } \\
\text { simulation }\end{array}$ & $\begin{array}{l}\text { Personalise risk } \\
\text { Information about others' } \\
\text { approval } \\
\text { Provide contingent rewards } \\
\text { Using imagery }\end{array}$ & $\begin{array}{l}\text { Encouragement to think about the } \\
\text { things that can happen when driving into } \\
\text { floodwater and when avoiding driving } \\
\text { into floodwater, including the risk and the } \\
\text { benefits; information about what important } \\
\text { others will think; outcome mental simulation } \\
\text { exercise }\end{array}$ & $\begin{array}{l}\text { Intention, attitudes, subjective norm, } \\
\text { perceived behavioural control, risk } \\
\text { perception, perceived severity, } \\
\text { perceived susceptibility, anticipated } \\
\text { regret }\end{array}$ \\
\hline $\begin{array}{l}\text { 6: } \\
\text { Conclusion }\end{array}$ & Cue altering & $\begin{array}{l}\text { Instructing that if ever in the situation to } \\
\text { remember goal }\end{array}$ & Barrier self-efficacy \\
\hline
\end{tabular}

Part 3 refers to the practice task which is designed to build imagery ability and does not relate to a target construct being assessed.

while driving their car and consider the steps they could take to avoid driving into the floodwater. Participants are then instructed to visualise these scenes independently for approximately two minutes. After the exercise is complete, the narrator advises the participant that the activity is over and requests that they note down the plan that they imagined in a response box below the video.

\section{Part 5: outcome imagery exercise}

Participants watch a video which guides them through an outcome imagery exercise. This involves imagining the outcomes that may occur if they drive into floodwater, followed by a range of outcomes that would occur if they do not drive into floodwater. After the exercise is complete, the narrator advises the participant that the activity is over and requests that they note down the things they imagined in the space below the video.

\section{Part 6: conclusion}

Following the final imagery exercise, participants watch a video thanking them for their attention and reminding them that if they are ever in the imagined situation, to remember their goal. The purpose of this is to create a cue that may be triggered by a situation similar to that imagined during the intervention.
Imagery fidelity

Fidelity of the imagery intervention will be assessed using four items modified from Knäuper et al. ${ }^{25}$ The items assess vividness, clarity, detail and ease of imagery on 7-point scales (eg, "How CLEAR was the mental image that you had of yourself avoiding driving through the floodwater?"). The 7-point scales vary for each item and are described in online supplementary material B.

\section{Control condition}

Participants in the control group will receive the part 1: education video and will then be directed to the T2 survey. This active control condition was chosen as the comparator to allow the effect of the imagery intervention to be examined above and beyond the effect of simply providing information about the behaviour.

\section{Outcomes}

Psychological measures

Participants will complete measures of psychological constructs from two social-cognitive theories: the theory of planned behaviour ${ }^{32}$ and the health action process approach. ${ }^{33}$ These theories were selected because they have used to identify the psychological determinants of a range of health and safety behaviours. ${ }^{34}{ }^{35}$ The psychological constructs will be measured on multi-item psychometric 
instruments developed using standardised guidelines. ${ }^{36}$ All scales measuring social-cognitive constructs except action planning and perceived behavioural control have been used in our previous study, ${ }^{19}$ exhibiting good internal consistency. While we discuss the behaviour in this study as 'driving into floodwater' because by nature there is no certainty around a driver making it through, we measure the behaviour using the wording 'driving through floodwater'. This is based on our prior qualitative work where participants most commonly described the behaviour in this way. ${ }^{14} 17$ The self-report psychological measures will be administered at T1, T2 and T3 (see online supplementary material B for all measures used in the study).

\section{Intention}

Intention to drive through floodwater will be measured using four items (eg, "I intend to drive through the floodwater"). Responses are provided on 7-point scales ( $1=$ strongly disagree and $7=$ strongly agree $)$. Change in intention is the primary outcome in the study. The following outcomes are secondary outcomes.

\section{Attitudes}

Attitudes towards driving through floodwater will be assessed using five items preceded by the common stem: "If I were to drive through the floodwater, it would be". Responses will be provided on semantic differential scales (eg, $1=$ bad and $7=$ good $)$.

\section{Subjective norm}

Subjective norm will be measured using five items prompting participants to rate the extent to which important others would want them to drive through floodwater and whether people similar to them would drive through (eg, "Most people who are important to me would approve of me driving through the floodwater"). Responses are provided on 7-point scales (1=strongly disagree and $7=$ strongly agree $)$.

\section{Perceived behavioural control}

Perceived behavioural control will be measured using three items assessing drivers' perceptions of their ability to control the behaviour (eg, "I have complete control over whether I drive through the floodwater"). Responses are provided on 7-point scales (1=strongly disagree and $7=$ strongly agree $)$.

\section{Risk perception}

Risk perception will be measured using two items (eg, "It would be risky for me to drive through the floodwater"). Responses are provided on 7-point scales (1=strongly disagree and $7=$ strongly agree $)$.

\section{Perceived susceptibility}

Perceived susceptibility will be measured using three items (eg, "My chances of having trouble if I drive through the floodwater are great"). Responses are provided on 7-point scales $(1=$ extremely unsusceptible and $7=$ extremely susceptible $)$.

\section{Perceived severity}

Perceived severity will be measured using two items (eg, "If I drive through the floodwater, the consequences would be..."). Responses are provided on 7-point scales ( $1=$ not at all severe and $7=$ extremelysevere).

\section{Anticipated regret}

Anticipated regret will be measured using three items (eg, "If I were to drive through the floodwater, I would feel regret"). Responses are provided on 7-point scales ( $1=$ strongly disagree and $7=$ strongly agree $)$.

\section{Barrier self-efficacy}

Barrier self-efficacy will be measured using nine items assessing drivers' confidence to avoid driving through floodwater (eg, "I am confident I can avoid driving through floodwaters in the future... even when the alternative route will take more time/is inconvenient"). Responses are provided on 7-point scales (1=strongly disagree and $7=$ strongly agree). The barrier self-efficacy items were developed using belief elicitation in our prior work ${ }^{17}$ with a sample of drivers who had avoided driving into floodwater when they encountered it on their route.

\section{Action planning}

Action planning will be measured using four items (eg, "I have made a plan regarding... How to avoid driving through floodwater"). Responses are provided on 7-point scales $(1=$ not at all true and $7=$ exactly true $)$.

\section{Covariates}

Consistent with the approach taken in our prior work, ${ }^{19}$ several demographic variables will be measured and used as covariates in the analyses. Goal intention and imagery ability will also be measured and used as covariates.

\section{Demographic and other background factors}

Demographic and background details are collected at T1 including: (i) sex $(0=$ maleand $1=$ female $)$; (ii) age (in years); (iii) relationship status $(0=$ not married and $1=$ married); (iv) education level ( $0=$ non-university and $1=$ university); (v) number of years driving; (vi) number of children and (vii) past frequency of driving through floodwater measured using a single item: "How often in the past 5 years have you driven through floodwater?" 'Floodwater' refers to a body of water covering land that is normally dry, with responses provided on a 7-point scale ( $1=$ neverand $7=$ very often $)$.

\section{Goal intention}

Following parts 1 and 2 of the intervention (or part 1 for the control group), participants will be asked a question to assess their willingness to form a goal to avoid driving into floodwater, "Now that you have heard some information about driving into floodwater, please indicate your agreement with the following statement: I am willing to form a goal to avoid driving through floodwater", responses provided on a 7-point scale (1=strongly disagree and to $7=$ strongly agree) . 


\section{Imagery ability}

Individual differences in imagery ability will be measured using a 10-item scale drawn from the International Personality Item Pool ${ }^{37}$ and designed to measure factor $\mathrm{V}$ (intellect and imagination) of Goldberg's Big-Five Factor Markers. ${ }^{38}$ Responses provided on 5-point scales $(1=$ very inaccurate and $5=$ very accurate $)$. For example, "Typically, I... Have a vivid imagination".

\section{Power analysis}

An a priori power analysis was conducted using $\mathrm{G}^{*}$ Power V.3.1 for an analysis of covariance (ANCOVA) model estimating fixed effects, main effects and interactions. The effect size was set to $\mathrm{f}=0.25$ (corresponding to the effect size of intention from preintervention to postintervention in the study by Hamilton $e t a l^{19}$ to detect a medium effect, with power set at 0.95 and alpha set at 0.01 (adjusted to protect from inflation of type I error rate due to multiple tests). Inclusion of nine covariates was also specified. The analysis yielded a total minimum required sample size of 289. We aim to recruit 460 participants at the baseline in order to meet our target sample size of 300 participants at the follow-up (allowing for up to $35 \%$ attrition). Recruitment of will cease when 460 participants have participated in the baseline.

\section{Statistical analysis}

Hypotheses will be tested using a series of mixed-model ANCOVAs. In the analyses, condition/group will be a between-participants variable; time (T1, T2, T3) will be a within-participants variable and the primary outcome (intention) and secondary outcomes (attitudes, subjective norm, perceived behavioural control, risk perception, perceived susceptibility, perceived severity, anticipated regret, barrier self-efficacy, action planning) will be separate dependent variables. Consistent with the approach taken in our prior work, ${ }^{19}$ demographic variables (sex, age, relationship status, educational level, number of years driving, number of children and past frequency of driving through floodwaters) will be included as covariates in the analyses. Mental imagery ability and goal intention will also be included as covariates. Where an ANCOVA indicates that there is a significant timexgroup interaction for any of the outcome variables, simple effects analyses using estimated marginal means will be conducted for that outcome. Specifically, we will compare within-group differences in the outcome between time points, and between-group differences in the outcome at each time point. Alpha will be set at 0.01 for all analyses (adjusted to protect from inflation of type I error rate due to multiple tests). Missing data will be imputed using the expectation-maximisation (E-M) algorithm.

\section{ETHICS AND DISSEMINATION}

We anticipate that the rigorous development and piloting process will ensure that no amendments to the protocol are required. However, if any amendments are required, they will be submitted as amendments to the Australian New Zealand Clinical Trials Registry record and reported in the final report of the study. We do not anticipate any risks greater than daily living to be involved with participation in this project, and no discomfort or adverse effects were reported by participants in the pilot study. However, participants are provided with the following information: 'should you experience any discomfort due to undertaking this survey, Lifeline (13 11 14) offers a free 24 hours telephone counselling service'.

\section{Informed consent}

Before being presented with the online baseline survey, participants are presented with the study information sheet. The information sheet indicates that proceeding to the next page and commencing participation will be considered consent to participate. Participants are also advised in the information sheet that they are free to cease participation at any time without comment or penalty (see online supplementary material $\mathrm{C}$ for informed consent materials).

\section{Confidentiality}

Participants will be recruited by a research panel provider and directed to an online Qualtrics survey to participate in the study. The research panel provider does not have access to participant responses, which are accessible only by members of the research team. The research team match responses using a code identifier, and the code identifier of those who complete the baseline are provided to the research panel provider to invite participants to complete the follow-up. The research team do not have access to the identities of participants.

\section{Data deposition}

Prior to publication of the results, data will be stored securely on Qualtrics and then the Griffith University Google Drive or OneDrive allocation and will be accessible only by authors KH and JJK. Once data collection is complete, data access will also be provided to author MSH. Given the restrictions on access to the data, a data monitoring committee will not be required. Following publication, deidentified data and statistical code will be made available on Open Science Framework.

\section{Dissemination}

The findings will be presented in the form of peer-reviewed journal articles and industry reports and will be presented at scientific conferences. The authors of this protocol will author publications arising from this trial. Media releases and public statements about the research will also be made to disseminate the findings to the general public. The findings will also be made available to participants if requested. Contact details and procedure for requesting the results will be made available in the study information sheet. Trial materials will be made available on Open Science Framework following publication of the results. 


\section{DISCUSSION}

The purpose of the current project is to evaluate the effectiveness of a theory-based intervention using implementation imagery to change drivers' intentions and beleifs to drive into floodwaters. The intervention will be delivered online using a survey tool containing a series of infographic videos that present intervention messages. Participants will be randomised to an intervention condition or a control condition. The videos presented to participants assigned to the intervention condition will comprise a series of educational messages about the risks of driving into floodwater and an exercise using implementation imagery. The videos presented to participants assigned to the control condition will comprise the education messages only. The primary outcome variable will be intentions to drive into floodwater, and secondary outcomes will be theory-based constructs identified as important determinants of intentions to drive into floodwater in our previous research.$^{14-19}$

Driving into floodwaters is a high-risk behaviour that can be fatal. Reducing drownings from risky aquatic activities during floods has been identified as a priority in the Australian Water Safety Strategy 2016-2020. ${ }^{12}$ Strategies that change drivers' behaviour in and around floodwater should be at the forefront of efforts to reduce drivers' propensity to drive into floodwaters. The present intervention examines whether a behavioural intervention will be effective in changing driver's intentions and beliefs to drive into floodwater. The intervention has been developed with a focus on effectiveness and potential feasibility. In terms of effectiveness, the intervention is based on psychological theories of attitudes, motivation and decision making, consistent with research indicating that effective behaviour change necessitates a fundamental understanding of behaviour, and that theory-based interventions can lead to more effective and efficient interventions. The intervention content is based on formative research identifying the determinants of risky behaviour when driving in floods and into floodwaters and is designed to target change in behaviour through change in those determinants. In terms of feasibility, the intervention has been designed to be delivered online and uses accessible infographic videos co-designed by researchers and stakeholders. The exercises contained in the videos are designed to be brief and cost-effective, reducing response burden and maximising the likelihood of engagement. If found to be effective, translation of the intervention into practice will be facilitated by these design features. The brief, online design means it could be incorporated in websites and disseminated through programmes such as learner driver education and during media campaigns during floods with minimal requirement for modification and at relatively low cost. It also paves the way for larger scale usability and feasibility trials.

The intervention will also make a contribution to theory. The current intervention adopts behaviour change strategies that target key theoretical determinants demonstrated to be related to risky driving behaviours in and around floodwater. We expect our test of the intervention to contribute to the evidence base of effective methods for changing behaviour. Such evidence is important in order to identify the components of behaviour change interventions that are effective in promoting behaviour change and contributes to more effective and optimally efficient interventions.

A key strength of the study is that it uses a sample with key demographic characteristics proportional to the distribution of flood-related transport deaths in the Australian population, ensuring results are generalisable. While the outcomes are an extensive range of psychological variables established to predict driving into floodwater, the unpredictable and infrequent occurrence of flood events means that examining the effect of our intervention on actual behaviour is not feasible within the timeframe of the study. A limitation of the study is therefore that the primary outcome is behavioural intentions rather than behaviour. The paucity of high-quality theory-based intervention research is not unique to driving into floodwater. For example, for another behaviour that carries high risk of drowning, alcohol use during aquatic activities, there has not been any intervention research in more than two decades. ${ }^{39}$ If effective, this type of intervention and mode of delivery may be developed and applied to other water safety behaviours with the potential to reduce drowning.

\section{Author affiliations}

${ }^{1}$ School of Applied Psychology, Griffith University, Brisbane, Queensland, Australia

${ }^{2}$ School of Psychology, Health Psychology and Behavioural Medicine Research Group, Curtin University, Perth, Western Australia, Australia

${ }^{3}$ Royal Life Saving Society - Australia, Broadway, New South Wales, Australia

${ }^{4}$ College of Public Health, Medical and Veterinary Sciences, James Cook University, Townsville, Queensland, Australia

${ }^{5}$ Faculty of Sport and Health Sciences, University of Jyväskylä, Jyväskylä, Finland

Acknowledgements The authors would like to thank Kevin Judge (Senior Technical Officer- Health, Griffith University) for recording and producing the videos that will deliver the intervention and control condition stimuli. The authors would also like to thank the drivers who assisted with the pilot of intervention materials.

Contributors KH, AEP and MSH conceptualised the study. KH, JJK and MSH designed the study. MSH will conduct the data analysis, and $\mathrm{KH}$ and MSH will interpret the findings. KH and JJK drafted the manuscript with revisions provided by AEP and MSH. All authors reviewed and approved the final manuscript as submitted.

Funding The study is supported by a Royal Life Saving Society—Australia and Menzies Health Institute Queensland industry partnership grant. MSH contribution was supported by a Finland Distinguished Professor (FiDiPro) award (Dnro 1801/31/2105) from Business Finland.

Competing interests AEP is employed by Royal Life Saving Society—Australia and affiliated with the College of Public Health, Medical and Veterinary Sciences, James Cook University, Townsville, Australia. Intervention development, data collection and analysis and interpretation of findings is conducted independent of author AEP and Royal Life Saving Society—Australia.

Patient consent for publication Not required.

Ethics approval The study protocol was approved by the Griffith University Human Research Ethics Committee (GU ref no: 2017/895).

Provenance and peer review Not commissioned; externally peer reviewed.

Open access This is an open access article distributed in accordance with the Creative Commons Attribution Non Commercial (CC BY-NC 4.0) license, which permits others to distribute, remix, adapt, build upon this work non-commercially, 
and license their derivative works on different terms, provided the original work is properly cited, appropriate credit is given, any changes made indicated, and the use is non-commercial. See: http://creativecommons.org/licenses/by-nc/4.0/.

\section{REFERENCES}

1. World Health OrganizationWorld Health Organization. Global report on drowning: Preventing a leading killer. Geneva, Switzerland, 2014. http://www.who.int/violence_injury_prevention/publications/ drowning_global_report/Final_report_full_web.pdf.

2. Di Mauro M, De Bruijn KM, Meloni M. Quantitative methods for estimating flood fatalities: towards the introduction of lossof-life estimation in the assessment of flood risk. Nat Hazards 2012;63:1083-113.

3. Jonkman S. Bierens J, Loss of life due to floods: General overview. Drowning. Berlin, Germany: Springer, 2014:957-65.

4. Peden AE, Franklin RC, Leggat $P$, et al. Causal pathways of flood related river drowning deaths in Australia. PLoS Curr 2017 May 18; Edition 1.

5. Peden AE, Franklin RC, Leggat P. The flood-related behaviour of river users in Australia. PLoS Curr 2018;10.

6. Peden AE, Franklin RC, Leggat PA. Fatal river drowning: the identification of research gaps through a systematic literature review. Inj Prev 2016;22:202-9.

7. Peden AE, Franklin RC, Mahony AJ, et al. Using a retrospective cross-sectional study to analyse unintentional fatal drowning in Australia: ICD-10 coding-based methodologies verses actual deaths. BMJ Open 2017;7:e019407.

8. National Oceanic and Atmospheric Administration. Turn around don't drown silver spring MD. 2011 http://www.nws.noaa.gov/os/water/ tadd/.

9. Queensland Floods Commission of Inquiry. Queensland floods commission of inquiry: final report. Brisbane, Australia, 2012. http:// www.floodcommission.qld.gov.au/_data/assets/pdf_file/0007/ 11698/QFCI-Final-Report-March-2012.pdf. (accessed 25 Apr 2018).

10. Peden $A E$, Franklin RC, Leggat PA. The hidden tragedy of rivers: a decade of unintentional fatal drowning in Australia. PLoS One 2016;11:e0160709.

11. Royal Life Saving Society - Australia. Royal Life Saving National Drowning Report 2016. Sydney, Australia, 2016. https://www. royallifesaving.com.au/_data/assets/pdf_file/0004/18085/RLS NDR2016_ReportLR.pdf.

12. Australian Water Safety Council. Australian Water Safety Strategy 2016-2020. Sydney, Australia: Australian Water Safety Council, 2016.

13. Royal Automobile Club of Queensland; RACQ. More than 100 floodwater rescues: If it is flooded, forget it. 2017 https://live.racq. com.au/2017/03/100-floodwater-rescues-flooded-forget/ (accessed 5 Jul 2017)

14. Hamilton K, Peden AE, Keech JJ, et al. Driving through floodwater: exploring driver decisions through the lived experience. International Journal of Disaster Risk Reduction 2018.

15. Hamilton K, Peden AE, Pearson M, et al. Stop there's water on the road! Identifying key beliefs guiding people's willingness to drive through flooded waterways. Saf Sci 2016;89:308-14.

16. Pearson $\mathrm{M}$, Hamilton $\mathrm{K}$. Investigating driver willingness to drive through flooded waterways. Accid Anal Prev 2014;72:382-90.

17. Hamilton K, Price S, Keech JJ, et al. Drivers' experiences during floods: Investigating the psychological influences underpinning decisions to avoid driving through floodwater. International Journal of Disaster Risk Reduction 2018;28:507-18.
18. Keech JJ, Smith SR, Peden AE, et al. The lived experience of rescuing people who have driven into floodwater: Understanding challenges and identifying areas for providing support. Health Promot J Austr 2018.

19. Hamilton K, Peden AE, Keech JJ, et al. Changing people's attitudes and beliefs toward driving through floodwaters: Evaluation of a video infographic. Transp Res Part F Traffic Psychol Behav 2018:53:50-60.

20. Johnson DP, Whisman MA. Gender differences in rumination: A meta-analysis. Pers Individ Dif 2013;55:367-74.

21. Hagger MS, Luszczynska A, de Wit J, et al. Implementation intention and planning interventions in Health Psychology: Recommendations from the Synergy Expert Group for research and practice. Psychol Health 2016;31:814-39.

22. Conroy D, Hagger MS. Imagery interventions in health behavior: $A$ meta-analysis. Health Psychol 2018;37:668-79.

23. Gollwitzer PM. Implementation intentions: Strong effects of simple plans. Am Psychol 1999;54:493-503.

24. Gollwitzer PM, Sheeran P. Implementation intentions and goal achievement: a meta-analysis of effects and processes. Advances in Experimental Social Psychology 2006;38:69-119.

25. Knäuper B, McCollam A, Rosen-Brown A, et al. Fruitful plans: adding targeted mental imagery to implementation intentions increases fruit consumption. Psychol Health 2011;26:601-17.

26. Hagger MS, Lonsdale A, Koka A, et al. An intervention to reduce alcohol consumption in undergraduate students using implementation intentions and mental simulations: a cross-national study. Int J Behav Med 2012;19:82-96.

27. Chan AW, Tetzlaff JM, Gøtzsche PC, et al. SPIRIT 2013 explanation and elaboration: guidance for protocols of clinical trials. BMJ 2013;346:e7586.

28. Chan AW, Tetzlaff JM, Altman DG, et al. SPIRIT 2013 statement: defining standard protocol items for clinical trials. Ann Intern Med 2013;158:200-7.

29. Kok G, Gottlieb NH, Peters GJ, et al. A taxonomy of behaviour change methods: an Intervention Mapping approach. Health Psychol Rev 2016;10:297-312.

30. Schroder HS, Dawood S, Yalch MM, et al. Evaluating the domain specificity of mental health-related mind-sets. Soc Psychol Personal Sci 2016;7:508-20.

31. Maniaci MR, Rogge RD. Caring about carelessness: Participant inattention and its effects on research. J Res Pers 2014;48:61-83.

32. Ajzen I. The theory of planned behavior. Organ Behav Hum Decis Process 1991;50:179-211.

33. Schwarzer R. Modeling health behavior change: how to predict and modify the adoption and maintenance of health behaviors. Appl Psychol 2008;57:1-29.

34. McEachan RRC, Conner M, Taylor NJ, et al. Prospective prediction of health-related behaviours with the Theory of Planned Behaviour: a meta-analysis. Health Psychol Rev 2011;5:97-144.

35. Zhang $\mathrm{C}$, Zhang $\mathrm{R}$, Schwarzer $\mathrm{R}$ et al. A meta-analysis of the health action process approach. Health Psychology 2019.

36. Ajzen I. Constructing a theory of planned behavior questionnaire, 2006.

37. Goldberg LR, broad-bandwidth A. public domain, personality inventory measuring the lower-level facets of several five-factor models. In: Mervielde I, Deary I, De Fruyt F, eds. Personality Psychology in Europe. Tilburg, The Netherlands: Tilburg University Press, 1999:7-28.

38. Goldberg LR. The development of markers for the Big-Five factor structure. Psychol Assess 1992;4:26-42.

39. Hamilton K, Keech JJ, Peden AE, et al. Alcohol use, aquatic injury, and unintentional drowning: A systematic literature review. Drug Alcohol Rev 2018;37:752-73. 